Irish Math. Soc. Bulletin

Number 79, Summer 2017, 75-85

ISSN 0791-5578

\title{
Composition operators between weighted Bergman spaces and weighted Banach spaces of holomorphic functions
}

\author{
ELKE WOLF
}

\begin{abstract}
An analytic self-map $\phi$ of the open unit disk $\mathbb{D}$ in the complex plane induces the so-called composition operator $C_{\phi}$ : $H(\mathbb{D}) \rightarrow H(\mathbb{D}), \quad f \mapsto f \circ \phi$, where $H(\mathbb{D})$ denotes the set of all analytic functions on $\mathbb{D}$. Motixated by [5] we analyze under which conditions on the weight $v$ a 1 conposition operators $C_{\phi}$ acting between the weighted Bergmran spare and the weighted Banach space of holomorphic functior bothgenerated by $v$ are bounded.
\end{abstract}

Let $\mathbb{D}$ denet the open unit dish in the comple prone $\mathbb{C}$ and $H(\mathbb{D})$ the space orall analytic fanctions on $\mathbb{D}$ endpred with the compactopen orology co. Mørecrer, let $\phi$ be ar apalytic self-map of $\mathbb{D}$. Sugha rosp induces throggh compos itiox the classical composition operator

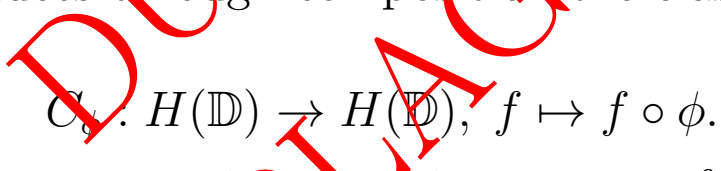

Composition operators acting onyarious spaces of analytic functions have been studied by many authors, since this kind of operator appears naturally in a ariety of problems, such as e.g. in the study of commutants of muliplication operators or the study of dynamical systems, see tha excellent monographs [8] and [17]. For a deep insight in the feent research on (weighted) composition operators we refer theveader to the following papers as well as the references therein: [4], [5], [6], [7], [11], [13], [14], [15], [16].

Let us now explain the setting in which we are interested. We say that a function $v: \mathbb{D} \rightarrow(0, \infty)$ is a weight if it is bounded and continuous. For a weight $v$ we consider the following spaces:

2010 Mathematics Subject Classification. 47B33, 47B38.

Key words and phrases. weighted Banach space of holomorphic functions, weighted Bergman space, composition operator.

Received on 18-8-2016; revised 10-1-2017. 
(1) The weighted Banach spaces of holomorphic functions defined by

$$
H_{v}^{\infty}:=\left\{f \in H(\mathbb{D}) ;\|f\|_{v}:=\sup _{z \in \mathbb{D}} v(z)|f(z)|<\infty\right\} .
$$

Endowed with the weighted sup-norm $\|\cdot\|_{v}$ this is a Banach space. These spaces arise naturally in several problems related to e.g. complex analysis, spectral theory, Fourier analysis, partial differential and convolution equations. Concrete examples may be found in [3]. Waighted Banach spaces of holomorphic functions have beenstuflied deeply in [2] and also in [1].

(2) The weighted Bergman speres given by

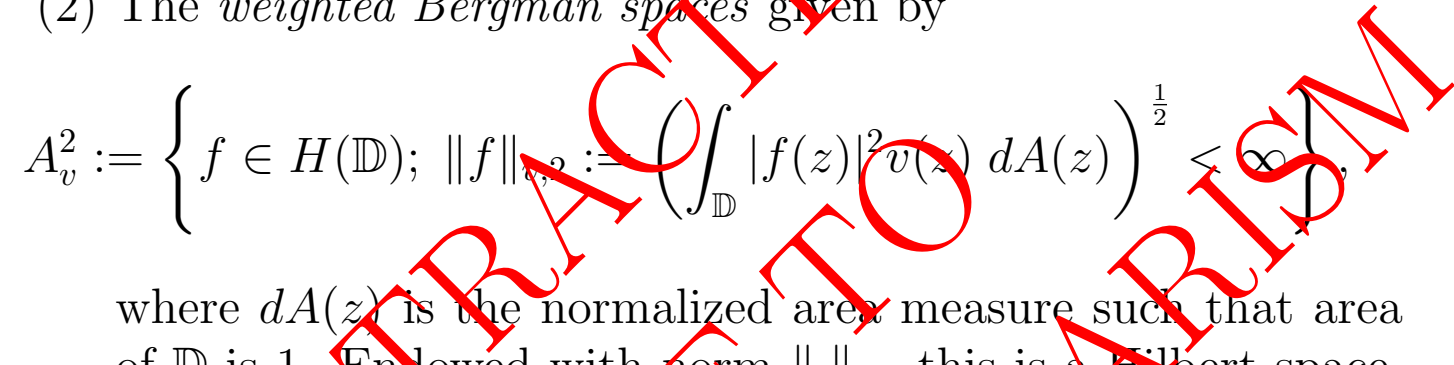
of $\mathbb{D}$ is 1 Endowed with orm $\|\cdot\|_{v, 2}$ this is anert space. An intreduction to Berghnanspaces is given in [10] and [9].

In [19] we chracterized the Domdedness de composition operators acting beween weighted Bergman spaces and weighted Banach spaces of holomorphic functions in terms gfthe involved weights as well as the symbols. In this articlewaronterested in the question, for which weights $v$ all composition yperators $C_{\phi}: A_{v}^{2} \rightarrow H_{v}^{\infty}$ are bounded.

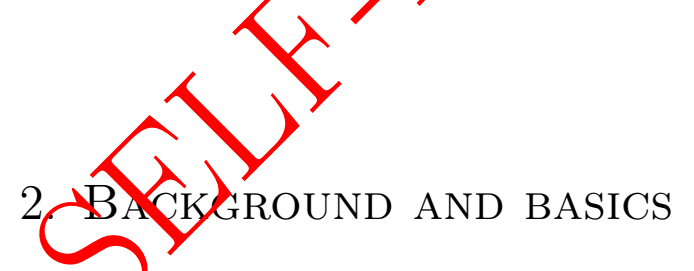

2.1. Theory of weights. In this part of the article we want to give some background information on the involved weights. A very important role play the so-called radial weights, i.e. weights which satisfy $v(z)=v(|z|)$ for every $z \in \mathbb{D}$. If additionally $\lim _{|z| \rightarrow 1} v(z)=0$ holds, we refer to them as typical weights. Examples include all the famous and popular weights, such as

(a) the standard weights $v(z)=(1-|z|)^{\alpha}, \alpha \geq 1$,

(b) the logarithmic weights $v(z)=(1-\log (1-|z|))^{\beta}, \beta>0$,

(c) the exponential weights $v(z)=e^{-\frac{1}{(1-|z|)^{\alpha}}}, \alpha \geq 1$. 
In [12] Lusky studied typical weights satisfying the following two conditions

$$
\text { (L1) } \inf _{n \in \mathbb{N}} \frac{v\left(1-2^{-n-1}\right)}{v\left(1-2^{-n}\right)}>0
$$

and

$$
\text { (L2) } \limsup _{n \rightarrow \infty} \frac{v\left(1-2^{-n-j}\right)}{v\left(1-2^{-n}\right)}<1 \text { for some } j \in \mathbb{N} .
$$

In fact, weights having $(L 1)$ and $(L 2)$ are normal weights in the sense of Shields and Williams, see [18]. The standard weights are normal weights, the logarithmic woights satisfy $(L 1)$, but not $(L 2)$ and the exponential weights athy neither $(L 1)$ nor $(L 2)$. In our context $(L 2)$ is not of interes twhile $(L 1)$ will play a secondary role. The formulation of results on wighted spaces often requires the socalled associated weights. Dor a weight $v$ its associated might is given by

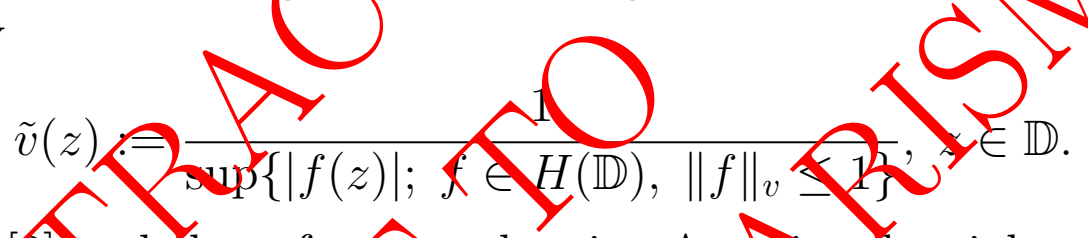

See e.g. (2) and the refernces therein. Assocrated weights are con-

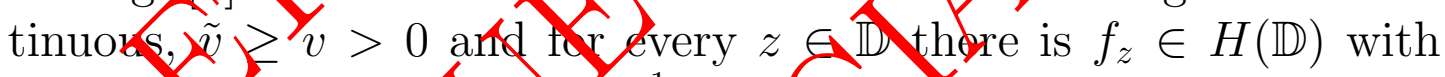

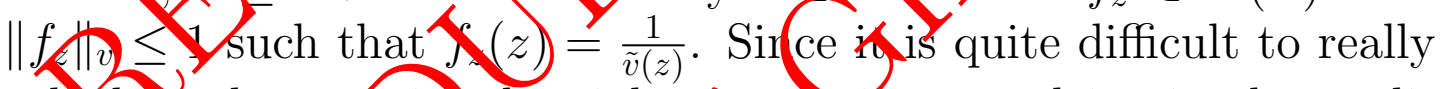
calc late the asspiated weight nore interested in simple conditions on the weight that ensure that $v$ and $\tilde{v}$ are equivalent weights, i.e. there is a constant $C \geq \mathrm{sych}$ that

$$
v(z) \leq \tilde{k}(z), C v(z) \text { for every } z \in \mathbb{D} \text {. }
$$

If $v$ and $\tilde{v}$ are equivarent, we say that $v$ is an essential weight. By [5] condition (LAT imp)ies the essentiality of $v$.

2.2. Setting. Mis section is devoted to the description of the setting we are wo king in. In the sequel we will consider weighted Bergman spaces generated by the following class of weights. Let $\nu$ be a holomorphic function on $\mathbb{D}$ that does not vanish and is decreasing as well as strictly positive on $[0,1)$. Moreover, we assume that $\lim _{r \rightarrow 1} \nu(r)=0$. Now, we define the weight as follows:

$$
v(z):=\nu(|z|) \text { for every } z \in \mathbb{D} \text {. }
$$

Obviously such weights are bounded, i.e. for every weight $v$ of this type we can find a constant $C>0$ such that $\sup _{z \in \mathbb{D}} v(z) \leq C$. Moreover, we assume additionally that $|\nu(z)| \geq \nu(|z|)$ for every 
$z \in \mathbb{D}$.

Now, we can write the weight $v$ in the following way

$$
v(z)=\min \{|g(\lambda z)|,|\lambda|=1\},
$$

where $g$ is a holomorphic function on $\mathbb{D}$. Since $\nu$ is a holomorphic function, we obviously can choose $g=\nu$. Then we arrive at

$$
\begin{array}{r}
\min \{|\nu(\lambda z)|,|\lambda|=1\}=\min \left\{\left|\nu\left(\lambda r e^{i \Theta}\right)\right|,|\lambda|=1\right\} \\
\leq\left|\nu\left(e^{-i \Theta} r e^{i \Theta}\right)\right|=|\nu(r)|=|\nu(|z|)|=v(z)
\end{array}
$$

for every $z \in \mathbb{D}$. Conversely, by hypothesis for every $\lambda \in \partial \mathbb{D}$ we obtain for every $z \in \mathbb{D}$

$$
|\nu(\lambda z)| \geq \nu(\mid \lambda z) \geq \nu(|z|)=v(z)
$$

Thus, the claim follows. The tandard, logarithmic and expenential weights can all be defined lirot that.

\subsection{Composition operyators botween /weighted Bengman} spaces and weightyd Banach spàces of helon orphic functions. In the sting of weighted Banach paces of holomorphic functions classical composition operator has been studied by Bonet, Domáq́ski, Lindstiömand Taskinenyiq [4] and [5]. Among other things they proved that in cas that $v$ and $w$ are arbitrary weights the boundedness of the operotor $\ell_{\phi}: H_{v}^{\infty} \rightarrow H_{w}^{\infty}$ is equivalent to

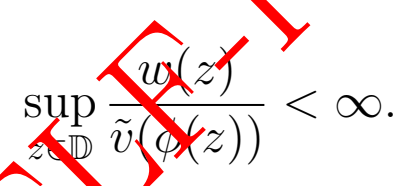

Moreover, they showed that satisfies condition $(L 1)$ if and only if every composition porator $C_{\phi}: H_{v}^{\infty} \rightarrow H_{v}^{\infty}$ is bounded.

This was the motiation to study the boundedness composition operators acting between weighted Bergman spaces and weighted Banach spaces of holomorphic functions. Doing this we obtain the following results which we need in the sequel. For the sake of understanding and completeness we give the proof here.

For $p \in \mathbb{D}$ let

$$
\alpha_{p}(z):=\frac{p-z}{1-\bar{p} z}, z \in \mathbb{D},
$$

be the Möbius transformation that interchanges $p$ and 0 . 
Lemma 2.1 ([20], Lemma 1). Let $v(z)=\nu(|z|)$ for every $z \in \mathbb{D}$ with $\nu \in H(\mathbb{D})$ be a weight as defined in Section 2.2. Then there is a constant $M>0$ such that

$$
|f(z)| \leq M \frac{\|f\|_{v, 2}}{\left(1-|z|^{2}\right) v(z)^{\frac{1}{2}}}
$$

for every $f \in A_{v}^{2}$.

Proof. As we have seen in Section 2.2 a weight as defined above may be written as

$$
v(z):=\min \{|g(\lambda z)|>1\} \text { for every } z \in \mathbb{D},
$$

where $g$ is a holomorphic fan on $\mathbb{D}$. In the sequel we will write $g_{\lambda}(z):=g(\lambda z)$ for ever $\in \mathbb{D}$. Now, fix $\lambda \in \mathbb{C}$ with $|\lambda|=1$. Moreover, let $p \in \mathbb{D}$ he arbitrary. Then, we consider thenap

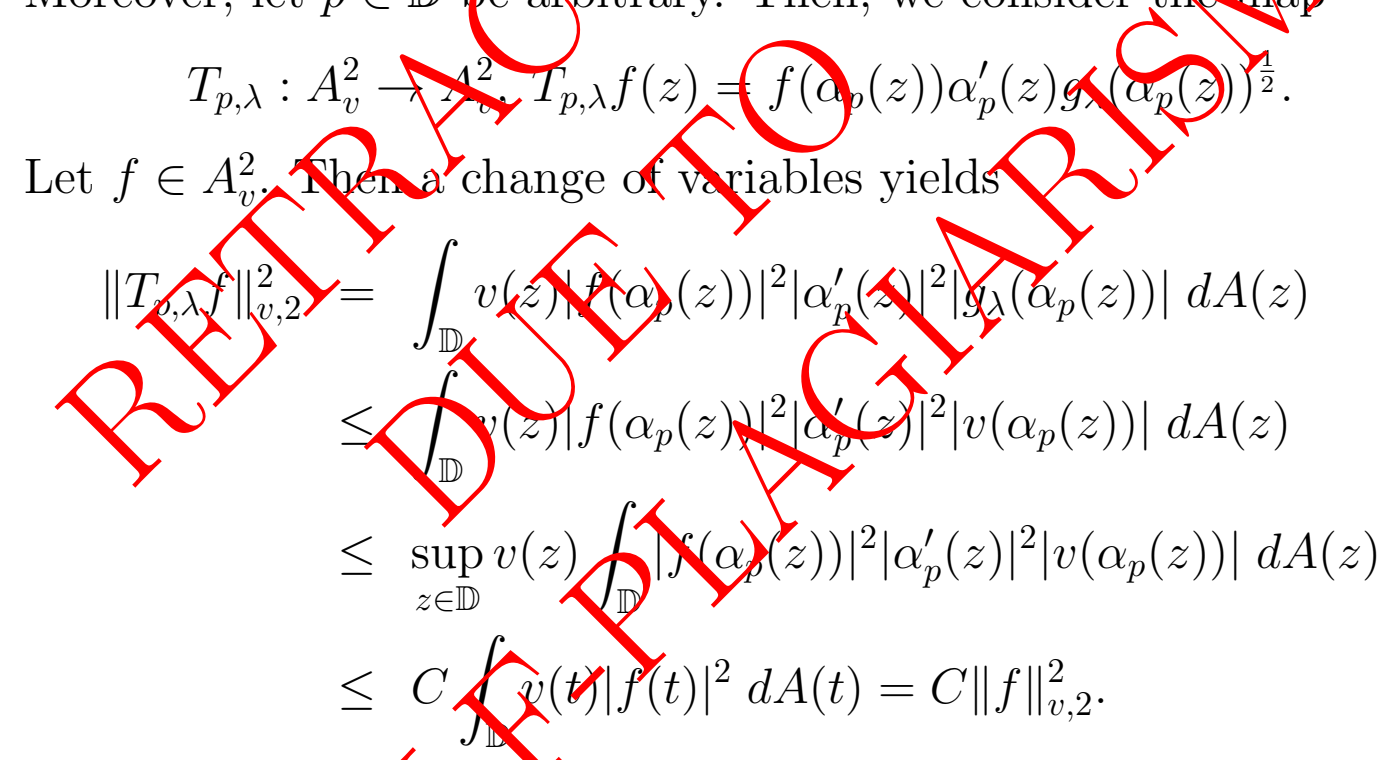

Next, put $h_{p, \lambda}(x): T_{p, \lambda} f(z)$ for every $z \in \mathbb{D}$. By the Mean Value Property we ontain

$$
v(0) \mid h_{p, \lambda}\left(0,\left.\right|^{2} \leq \int_{\mathbb{D}} v(z)\left|h_{p, \lambda}(z)\right|^{2} d A(z) \leq\left\|h_{p, \lambda}\right\|_{v, 2}^{2} \leq C\|f\|_{v, 2}^{2} .\right.
$$

Since $\lambda$ was arbitrary, we obtain

$$
v(0)|f(p)|^{2}\left(1-|p|^{2}\right) v(p)^{\frac{1}{2}} \leq C\|f\|_{v, 2}^{2} .
$$

Finally,

$$
|f(p)| \leq M \frac{\|f\|_{v, 2}}{\left(1-|p|^{2}\right) v(p)^{\frac{1}{2}}}<\infty .
$$


The following result is obtained by using the previous lemma and following exactly the proof of [19] Theorem 2.2. Again, for a better understanding we give the proof.

Theorem 2.2. Let $v(z)=\nu(|z|)$ for every $z \in \mathbb{D}$ with $\nu \in H(\mathbb{D})$ be a weight as defined in Section 2.2. Then the operator $C_{\phi}: A_{v}^{2} \rightarrow H_{v}^{\infty}$ is bounded if and only if

$$
\sup _{z \in \mathbb{D}} \frac{v(z)}{\left(1-|\phi(z)|^{2}\right) v(\phi(z))^{\frac{1}{2}}}<\infty .
$$

Proof. First, we assume that (2) holds. Applying Lemma 2.1 for every $f \in A_{v}^{2}$ we have

$$
|f(z)| \leq \frac{\|\left. f\right|_{v, 2}}{\left(1-|z|^{2}\right) v(z)^{\frac{1}{2}}}
$$

for every $z \in \mathbb{D}$. Thus, for erocy $f \in A_{y}^{2}$.

$$
\text { for every } z \in \mathbb{D} \text {. Thus, for erowy } f \in A_{i}^{2}
$$

Conversely, lect $p \in \mathbb{D}$ be fixod. Then there is $f_{p}^{2} \in H_{v}^{\infty},\left\|f_{p}^{2}\right\|_{v} \leq 1$ with $\left|f_{p}^{2}(p)\right|=\frac{1}{\tilde{v}(p)}$. Now, put

$$
g_{p}(z):=f_{p}(z) \alpha_{p}^{\prime}(z) \text { for exry } z \in \mathbb{D} \text {. }
$$

Changing variables we obtain

$$
\left\|g_{p}\right\|_{v, 2}^{2}=\int_{\mathbb{D}}\left|g_{p}(z)\right|^{2} v(z d \mathbf{N}(z))^{\prime}=\int_{\mathbb{D}}\left|f_{p}(z)\right|^{2}\left|\alpha_{p}^{\prime}(z)\right|^{2} v(z) d A(z)
$$

Next, we assume to the contrary that there is a sequence $\left(z_{n}\right)_{n} \subset \mathbb{D}$ such that $\left|\phi\left(z_{n}\right)\right| \rightarrow 1$ and

$$
\frac{v\left(z_{n}\right)}{\left(1-\left|\phi\left(z_{n}\right)\right|^{2}\right) v\left(\phi\left(z_{n}\right)\right)^{\frac{1}{2}}} \geq n \text { for every } n \in \mathbb{N} .
$$

Now, we consider

$$
g_{n}(z):=g_{\phi\left(z_{n}\right)}(z) \text { for every } z \in \mathbb{D} \text { and every } n \in \mathbb{N}
$$


as defined above. Then $\left(g_{n}\right)_{n}$ is contained in the closed unit ball of $A_{v}^{2}$ and we can find a constant $c>0$ such that

$$
c \geq v\left(z_{n}\right)\left|g_{n}\left(\phi\left(z_{n}\right)\right)\right|=\frac{v\left(z_{n}\right)}{\left(1-\left|\phi\left(z_{n}\right)\right|^{2}\right) v\left(\phi\left(z_{n}\right)\right)^{\frac{1}{2}}} \geq n
$$

for every $n \in \mathbb{N}$. Since we know that under the given assumptions we have $v=\tilde{v}$ this is a contradiction.

Having now characterized the boundedness of the composition operator acting between $A_{v}^{2}$ and $H_{v}^{\infty}$ we take the second result of Bonet, Domański, Lindström and Taskinen as a motivation to ask the question: For which weights $v$ are al dpenators $C_{\phi}: A_{v}^{2} \rightarrow H_{v}^{\infty}$ bounded?

\section{ZESULTS}

Lemma 3.1. Let $v(2=y(|z|)$ for every $z \in \mathbb{D}$ with $<$ \&

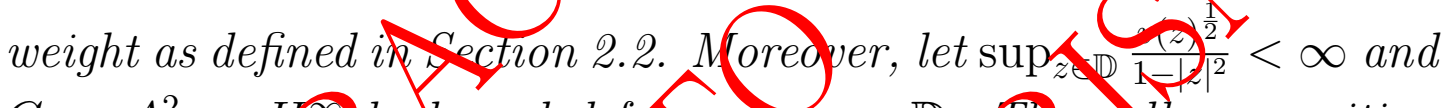
$C_{\alpha_{p}}: A_{v}^{2} \rightarrow$ be bounded forevery $p \in \mathbb{D}$. Then aul composition operators $\widehat{\hat{\beta}_{0}}: \mathbf{X}^{2} \rightarrow H_{v}^{\infty}$ gre bounded.

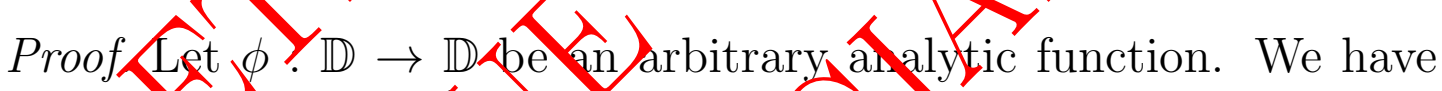
to shpw that $C_{\phi}: A_{v} \rightarrow H_{v}^{\infty}$ is bounde Now, $\phi=\alpha_{p} \circ \psi$ where $p=\varnothing(-), \psi=\alpha$, $\psi$ and $\psi(0)=\phi$ sined $\psi(0)=0$, by the Schwarz Lemma we obtain hat $|\psi(z)| \leq|z|$. Frence we get

$$
\sup _{z \in \mathbb{D}} \frac{v(z)}{\left(1-|\psi(z)|^{2}\right) v(\psi(z))^{\frac{1}{2}}} \sup _{z \in \mathbb{D}} \frac{v(z)}{\left(1-|z|^{2}\right) v(z)^{\frac{1}{2}}}=\sup _{z \in \mathbb{D}} \frac{v(z)^{\frac{1}{2}}}{\left(1-|z|^{2}\right)}
$$

$<\infty$. Thus, $C_{\psi}$ is bounded. Finally, we can conclude that $C_{\phi}$ is bounded since it 1s a composition of bounded operators.

The proof phe following theorem is inspired by [5].

Theorem 3.2. Let $v(z)=\nu(|z|)$ for every $z \in \mathbb{D}$ with $\nu \in H(\mathbb{D})$ be a weight as defined in Section 2.2. Moreover, let $\sup _{z \in \mathbb{D}} \frac{v(z)^{\frac{1}{2}}}{1-|z|^{2}}<\infty$. Then the composition operator $C_{\phi}: A_{v}^{2} \rightarrow H_{v}^{\infty}$ is bounded for every analytic self-map $\phi$ of $\mathbb{D}$ if and only if

$$
\inf _{n \in \mathbb{N}} \frac{\left(2^{-n}-2^{-2 n-2}\right) v\left(1-2^{-n-1}\right)^{\frac{1}{2}}}{v\left(1-2^{-n}\right)}>0 .
$$

Proof. By Lemma 3.1 we have to show that condition (3) holds if and only if $C_{\alpha_{p}}: A_{v}^{2} \rightarrow H_{v}^{\infty}$ is bounded for every $p \in \mathbb{D}$. 
First, let each $C_{\alpha_{p}}: A_{v}^{2} \rightarrow H_{v}^{\infty}$ be bounded. Then we have that for every $p \in \mathbb{D}$ there is $M_{p}>0$ such that

$$
v(z) \leq M_{p} v\left(\alpha_{p}(z)\right)^{\frac{1}{2}}\left(1-\left|\alpha_{p}(z)\right|^{2}\right) \text { for every } z \in \mathbb{D} .
$$

Since $\sup _{|z|=r}\left|\alpha_{p}(z)\right|=\frac{|p|+r}{1+|p| r}$ it follows that

$v(z) \leq M_{p} v\left(\frac{|p|+r}{1+|p| r}\right)^{\frac{1}{2}}\left(1-\left(\frac{|p|+r}{1+|p| r}\right)^{2}\right)$ for all $|z|=r$. Let $l(r)=$ $v(1-r)^{\frac{1}{2}}\left(1-(1-r)^{2}\right)$ and $s=1-r$. Now, since $1-\frac{|p|+1-s}{1+|p|(1-s)}=$ $\frac{s(1-|p|)}{1+|p|-|p| s}$, for $s<\frac{1}{2}$ we obtain

$$
l\left(s \frac{1-|p|}{1+|p|}\right) \leq l\left(1-\frac{|p|+1 \hat{s}}{1+\mid p(\hat{1}-\hat{s})}\right) \leq l\left(s \frac{1-|p|}{1-\frac{|p|}{2}}\right)
$$

Next, choose $p=\frac{2}{5}$ and find $M>0$ and $s_{0}>0$ such that $v(1-s) \leq M l\left(\frac{s}{2}\right)=\operatorname{Mar}\left(-\frac{s}{2}\right)^{\frac{1}{2}}$ Hence the claim forows. Conversely we assume that holds. Then as deined above has the propery there aren $>0$ and $t_{0} \in 10$, w with

$$
v(1-t) \leq M l\left(\frac{t}{2}\right) \text { for ayll } t \geq t_{0}
$$

Hence, for any $c<\infty$ we find $n \in \mathbb{N}$ such that $c<2^{n}$ and thus $l(t) \leq M^{n} l\left(\frac{t}{c}\right)$. We take $c=\frac{1+|p|}{|p|}$. By the first inequality in $(4)$ for all $p \in \mathbb{D}$ there is $M_{p}>0$ unch trat

$$
v(1 \odot t) \operatorname{RI}_{p} l\left(1-\frac{|p|+1-t}{1+|p|(1-t)}\right)
$$

for all $t>t_{0}$. Clearly this implies that for all $p \in \mathbb{D}$ there exists $M_{p}>$ 0 such that for every $|z|=r$ we have that $v(z)<M_{p} v\left(\alpha_{p}(z)\right)^{\frac{1}{2}}(1-$ $\left.\left|\alpha_{p}(z)\right|^{2}\right)$.

Example 3.3. (a) Let $v(z)=(1-|z|)^{n}, n \geq 2$. Then all composition operators $C_{\phi}: A_{v}^{2} \rightarrow H_{v}^{\infty}$ are bounded. To prove this we have to show that the weight $v$ satisfies the following conditions

(1) $\sup _{z \in \mathbb{D}} \frac{v(z)^{\frac{1}{2}}}{1-|z|^{2}}<\infty$, 
(2) $\inf _{k \in \mathbb{N}} \frac{\left(2^{-k}-2^{-2 k-2}\right) v\left(1-2^{-k-1}\right)^{\frac{1}{2}}}{v\left(1-2^{-k}\right)}>0$.

Indeed,

$$
\begin{gathered}
\sup _{z \in \mathbb{D}} \frac{v(z)^{\frac{1}{2}}}{1-|z|^{2}}=\sup _{z \in \mathbb{D}} \frac{(1-|z|)^{\frac{n}{2}}}{(1-|z|)(1+|z|)}=\sup _{z \in \mathbb{D}} \frac{(1-|z|)^{\frac{n-2}{2}}}{(1+|z|)} \\
\leq \sup _{z \in \mathbb{D}}(1-|z|)^{\frac{n-2}{2}}<\infty \text { since } n \geq 2 . \text { Moreover, } \\
\inf _{k \in \mathbb{N}} \frac{\left(2^{-k}-2^{-2 k-2}\right) v\left(1-2^{-k-1}\right)^{\frac{1}{2}}}{v\left(1-2^{-k}\right)}=\inf _{k \in \mathbb{N}}\left(2^{-k}-2^{-2 k-2}\right) 2^{\frac{1}{2}(k n-n)}
\end{gathered}
$$
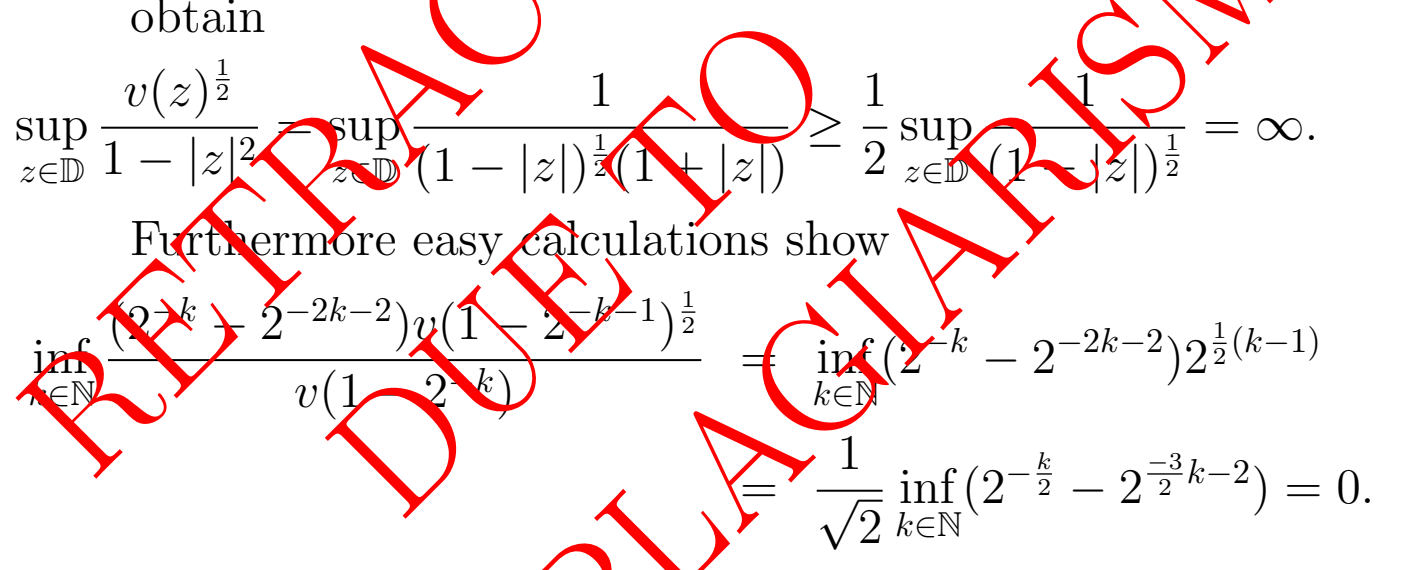

Hence, in this case there exists a composition operator $C_{\phi}$ : $A_{v}^{2} \rightarrow H_{v}^{\infty}$ that is notbounded. For example, the operator $C_{\phi}$ generated $\lambda_{\text {. }}$ the map $\phi(z)=z$ for every $z \in \mathbb{D}$ is not bounded, srice)

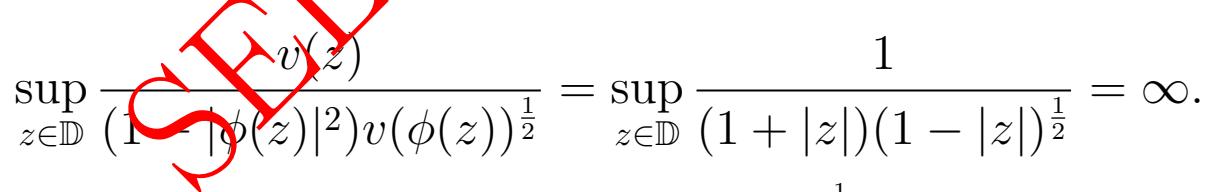

(c) The exponential weights $v(z)=e^{-\frac{1}{(1-|z|)^{n}}}, n>0$, satisfy condition (1), but not condition (2). First, we get

$$
\sup _{z \in \mathbb{D}} \frac{v(z)^{\frac{1}{2}}}{1-|z|^{2}}=\sup _{z \in \mathbb{D}} \frac{e^{-\frac{1}{2(1-|z|)^{n}}}}{1-|z|}<\infty .
$$

It follows, that (1) is fulfilled. Now,

$$
\inf _{k \in \mathbb{N}} \frac{\left(2^{-k}-2^{-2 k-2}\right) v\left(1-2^{-k-1}\right)^{\frac{1}{2}}}{v\left(1-2^{-k}\right)}=\inf _{k \in \mathbb{N}}\left(2^{-k}-2^{-2 k-2}\right) \frac{e^{-2^{k} n}}{e^{-2^{k} n}}=0 .
$$


Thus, (2) is not satisfied. There must be a composition operator $C_{\phi}: A_{v}^{2} \rightarrow H_{v}^{\infty}$ that is not bounded.

(d) The logarithmic weights $v(z)=\frac{1}{(1-\log (1-|z|))^{n}}, n>0$, neither satisfy (1) nor (2). Indeed,

$$
\sup _{z \in \mathbb{D}} \frac{v(z)^{\frac{1}{2}}}{1-|z|^{2}}=\sup _{z \in \mathbb{D}} \frac{1}{\left(1-|z|^{2}\right)(1-\log (1-|z|))^{\frac{n}{2}}}=\infty
$$

and

$$
\inf _{k \in \mathbb{N}} \frac{\left(2^{-k}-2^{-2 k-2}\right) v\left(1-2^{-k-1}\right)^{\frac{1}{2}}}{v\left(1-2^{-k}\right)}=\inf _{k \in \mathbb{N}}\left(2^{-k} 2^{-2 k-2}\right) \frac{\left(1-\log 2^{-k}\right)^{n}}{\left(1-\log 2^{-k-1}\right)^{\frac{n}{2}}}
$$

$=0$. With the criteria above wa gannot decide, whether all composition operators $C_{\infty} A_{v}^{2}$ A $H_{v}^{\infty}$ are bounded or ngt.

But, again selecting $\phi(z-z$ we see that

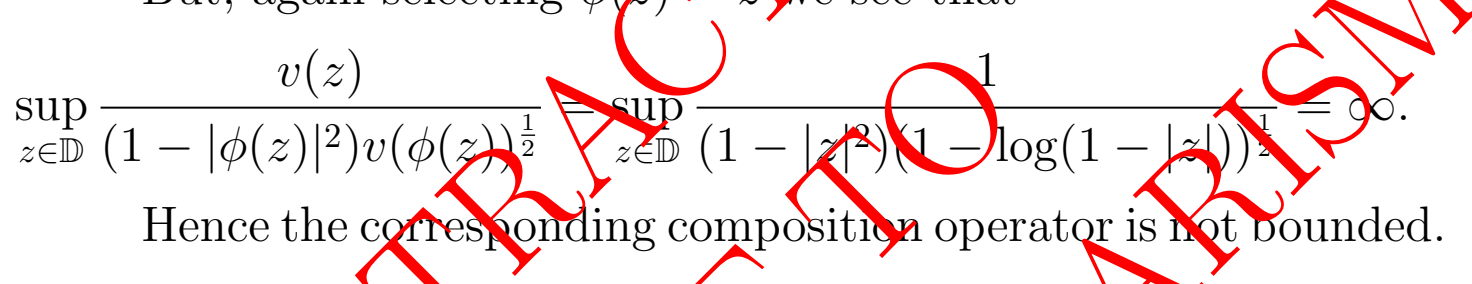

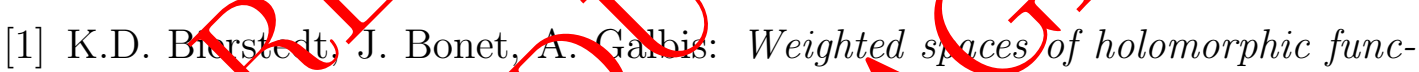
tions on buynced domain, Mighigan Math. J.4 4 , no. 2, (1993), 271-297.

[2] K.D. Bierstedt, J. Bonet, J. Naskinen: Aksociated weights and spaces of holomorphic functions, Studia Math. 127 no. Q (1998), 137-168.

[3] K. D. Bierstedt, R. Meise, W.H. Sunmers: A projective description of weighted inductive limits, Trang Am. Yath. Soc. 272, no. 1, (1982), 107160.

[4] J. Bonet, P. Domański, M. Linds cöm: Essential norm and weak compactness of composition operator 0 we ighted Banach spaces of analytic functions, Canad. Math. Bull. 42 ne. Q, (1999), 139-148.

[5] J. Bonet, P. Domaniski, L Lindström: J. Taskinen, Composition operators between weighted Bunach spaces of analytic functions, J. Austral. Math. Soc. (Series A) 64 (1998), I01-118.

[6] J. Bonet, M. Lindström, E. Wolf: Differences of composition operators between weighted Banach spaces of holomorphic functions, J. Aust. Math. Soc. 84 (2008), no. 1, 9-20.

[7] M.D. Contreras, A.G. Hernández-Díaz: Weighted composition operators in weighted Banach spaces of analytic functions, J. Austral. Math. Soc. (Series A) 69 (2000), 41-60.

[8] C. Cowen, B. MacCluer: Composition Operators on Spaces of Analytic Functions, CRC Press, Boca Raton, 1995.

[9] P. Duren, A. Schuster: Bergman spaces, Mathematical Surveys and Monographs 100, American Mathematical Society, Providence, RI, 2004. 
[10] H. Hedenmalm, B. Korenblum, K. Zhu: Theory of Bergman spaces, Graduate Texts in Mathematics 199, Springer-Verlag, New York, 2000.

[11] T. Kriete, B. MacCluer: Composition operators on large weighted Bergman spaces, Indiana Univ. Math. J. 41 (1992), no. 3, 755-788.

[12] W. Lusky: On weighted spaces of harmonic and holomorphic functions, J. London Math. Soc. (2) 51 (1995), no. 2, 309-320.

[13] J. Moorhouse, Compact differences of composition operators, J. Funct. Anal. 219 (2005), no. 1, 70-92.

[14] B. MacCluer, S. Ohno, R. Zhao: Topological structure of the space of composition operators on $H^{\infty}$, Integral equations Operator Theory 40 (2001), no. 4, 481-494.

[15] P. Nieminen: Compact differences of composition operators on Bloch and Lipschitz spaces, Comput. Metho ss. Funct. Theory 7 (2007), no. 2, 325-344.

[16] N. Palmberg: Weighted compostition operators with closed range, Bull. Austral. Math. Soc. 75 (2007) 20. 3. 31-354.

[17] J.H. Shapiro: Compogition Operators and Classical Functkn Theory, Springer, 1993.

[18] A.L. Shields, D.L. Willianks: Bounded projetions, duality mondipliers in spaces of harmopic furctions, J. Reine Angew. Math. 290/200 1978), 256279.

[19] E. Wolf: Leightedrcomposition Cnerators between wenghtee Bergman spaces and weighted Banach spaces of holo,norphic function Rev. Mat. Complut. 21 (2008), no. 2, 475-480 2

[20] E. Wolf; Composition folved by differentî tion between weighted Bergman spacs ond weighte Banach spaces of hotomorphic functions, Bull. Acad. Sfintse Repub. Mord Mat. 2014, no. 2, 29-35.

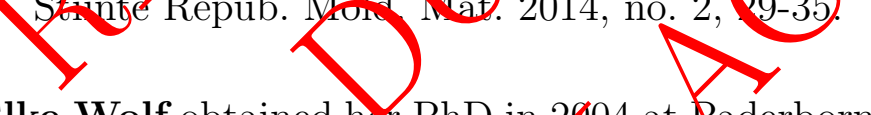

Elke Wolf obtained her PhD in 2004 at Paderborn and her Habilitation in 2010 at Trier. Currently she works at e dnitersity of Paderborn. In her private life she enjoys running, cycling and rading.

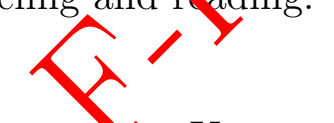

Institut für MÁnemałk, Universität PAderborn, Warburger Str. 100, 33098 PADERBORy/GERMANY

E-mail addras: Picl te@math.uni-paderborn.de

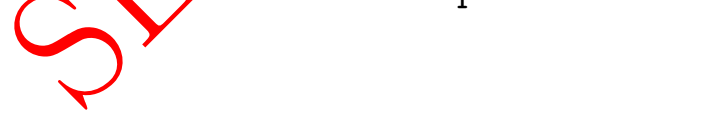

